\title{
Klinische Forschung und wissenschaftlich fundierte Weiterbildung
}

Liebe Leserinnen und Leser, sehr herzlich begrüße ich Sie zu diesem dritten Heft der neuen DGIM-Gesellschaftsausgaben. Auch wenn wir noch ganz am Anfang dieser neuen Heftreihe stehen, kann man doch jetzt schon sagen, dass die Entscheidung für dieses neue Format sich sehr bewährt hat. Die grundlegende Idee, die Innere Medizin in all ihren Facetten darzustellen und die Schwerpunkte der Inneren Medizin im Kontext der gesamten Inneren Medizin zu sehen, wird der Komplexität unseres Faches sehr gerecht. Viele von Ihnen waren sicher vor wenigen Wochen auf dem 117. Kongress der DGIM in Wiesbaden; wir hoffen sehr, dass unser Anliegen, die Innere Medizin als Gesamtheit darzustellen, auch dort gute Resonanz gefunden hat.

Ein wesentliches Anliegen der Gesellschaft ist es, wissenschaftlich begründete Weiterbildung und klinische Forschung zu fördern. Dies sagt sich einfacher, als es in der täglichen Praxis gelebt werden kann. Die Balance aus klinischer und forschender Tätigkeit aufrecht zu halten, ist alles andere als eine leichte Aufgabe. Wir sind der festen Überzeugung, dass fachlich herausragend gestaltete Artikel in den vorliegenden Heften auch dazu beitragen, die Förderung von Wissenschaft und Forschung auf dem Gesamtgebiet der Inneren Medizin voranzubringen. Die Deutsche Gesellschaft für Innere Medizin hat diese Aufgabe zu ihrer wichtigsten gemacht und versucht durch unterschiedliche Herangehensweisen die Position der forschenden Ärzte und damit der klinischen Forschung in der Inneren Medizin zu stärken.

Ich will an dieser Stelle nur einige der Möglichkeiten der Deutschen Gesellschaft für Innere Medizin nennen. So hat sich hinsichtlich der Nachwuchsförderung die DGIM und ihre Kommission zur „Wissenschaft und Nachwuchsförderung“ sehr klar für eine Gleichstellung (auch finanzielle Gleichstellung) grundlagenwissenschaftlich arbeitender Ärzte mit ihren klinischen Kollegen ausgesprochen; die wissenschaftliche Arbeit bedarf ganz eindeutig derselben Wertschätzung wie die klinische Arbeit. Auch für die Zukunft wird es eine wesentliche Aufgabe sein, Komplementarität und gegenseitige Abhängigkeit beider Ebenen klarzustellen. Klinische Forschung bedeutet eben auch translationale Forschung in beide Richtungen: „from bench to bedside“ ebenso wie „from bedside to bench“. Für die DGIM ist es selbstverständlich, in diesem Zusammenhang auch alles für eine Förderung des weiblichen Nachwuchses in der Inneren Medizin zu tun.
Ein weiteres bedeutsames Element in der Förderung des wissenschaftlichen Nachwuchses ist die Integration von Forschungsergebnissen junger Wissenschaftler in unseren Jahreskongress, aber natürlich auch in die Publikationsorgane der DGIM. Wir haben versucht, auf dem zurückliegenden Kongress dieses Konzept sehr dezidiert umzusetzen; Beispiele hierfür sind die konkurrenzfreie Postersitzung, die Young-InvestigatorAward-Auswahlsitzung wie auch die Preisverleihung.

Klinische Forschung in der Inneren Medizin wird und kann aber nur dann erfolgreich sein, wenn die Innere Medizin als Gesamtheit, aber auch ihre Schwerpunkte unvermindert gestärkt werden und adäquat an Universitätskliniken und Schwerpunktkrankenhäusern repräsentiert sind. Der Einsatz für eine sinnvolle Verteilung der Schwerpunkte und eine klare Vermeidung von Unterrepräsentanz ist daher auch eine entscheidende Aufgabe der DGIM, die zum Beispiel in der Kommission „Struktur - Krankenversorgung“ verfolgt werden.

Schließlich ist ein ganz wesentliches Ziel in diesem Zusammenhang die enge Zusammenarbeit mit Schwerpunktgesellschaften der Inneren Medizin, aber auch mit den Regionalgesellschaften, um möglichst alle internistisch arbeitenden Kollegen in Deutschland für die Ziele der DGIM zu erreichen und hierfür zu begeistern.

Das Erreichen dieser Ziele ist ein dynamischer Prozess und wird auch in den nächsten Jahren eine kontinuierliche Anpassung der Strukturen fordern. Die DGIM-Gesellschaftsausgaben der Deutschen Medizinischen Wochenschrift sind sicher hoch geeignet, um diese Anliegen nicht nur zu unterstützen, sondern auch um sie umzusetzen.

Ihr

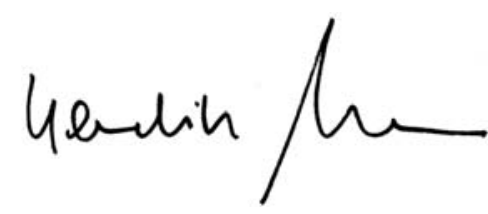

Prof. Dr. med. Hendrik Lehnert

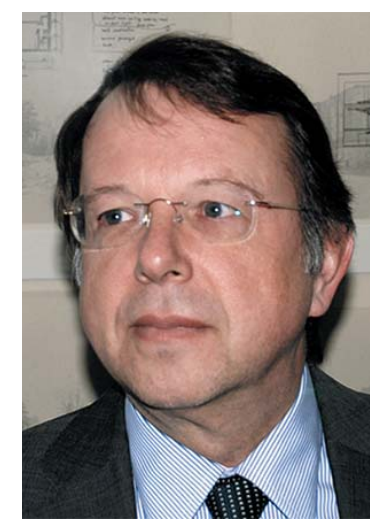

H. Lehnert

Institut

Medizinische Klinik I,

Universitätsklinikum SchleswigHolstein, Campus Lübeck

Bibliografie

DOI 10.1055/s-0031-1280529 Dtsch Med Wochenschr 2011 136: 1161 - (C) Georg Thieme Verlag KG Stuttgart - New York . ISSN 0012-0472

Korrespondenz Prof. Dr. Hendrik Lehnert Medizinische Klinik 1 Universitätsklinikum Schleswig-Holstein Campus Lübeck Ratzeburger Allee 160 (Haus 40) 23538 Lübeck Tel. 0451/500-2306 Fax 0451/500-3339 